\title{
Development of a human three-dimensional organotypic skin-melanoma spheroid model for in vitro drug testing
}

\author{
H Vörsmann ${ }^{1}$, F Groeber ${ }^{2}$, H Walles ${ }^{3}$, S Busch ${ }^{4}$, S Beissert ${ }^{5}$, H Walczak ${ }^{6}$ and D Kulms ${ }^{\star, 1,5}$
}

Despite remarkable efforts, metastatic melanoma (MM) still presents with significant mortality. Recently, mono-chemotherapies are increasingly replenished by more cancer-specific combination therapies involving death ligands and drugs interfering with cell signaling. Still, MM remains a fatal disease because tumors rapidly develop resistance to novel therapies thereby regaining tumorigenic capacity. Although genetically engineered mouse models for MM have been developed, at present no model is available that reliably mimics the human disease and is suitable for studying mechanisms of therapeutic obstacles including cell death resistance. To improve the increasing requests on new therapeutic alternatives, reliable human screening models are demanded that translate the findings from basic cellular research into clinical applications. By developing an organotypic full skin equivalent, harboring melanoma tumor spheroids of defined sizes we have invented a cell-based model that recapitulates both the 3D organization and multicellular complexity of an organ/tumor in vivo but at the same time accommodates systematic experimental intervention. By extending our previous findings on melanoma cell sensitization toward TRAIL (tumor necrosis factor-related apoptosis-inducing ligand) by co-application of sublethal doses of ultraviolet-B radiation (UVB) or cisplatin, we show significant differences in the therapeutical outcome to exist between regular two-dimensional (2D) and complex in vivo-like 3D models. Of note, while both treatment combinations killed the same cancer cell lines in 2D culture, skin equivalent-embedded melanoma spheroids are potently killed by TRAIL + cisplatin treatment but remain almost unaffected by the TRAIL + UVB combination. Consequently, we have established an organotypic human skin-melanoma model that will facilitate efforts to improve therapeutic outcomes for malignant melanoma by providing a platform for the investigation of cytotoxic treatments and tailored therapies in a more physiological setting.

Cell Death and Disease (2013) 4, e719; doi:10.1038/cddis.2013.249; published online 11 July 2013

Subject Category: Cancer

Malignant melanoma is a highly aggressive cancer with rapidly increasing incidence. The prognosis for patients with metastatic melanoma (MM) is particularly poor with stage IV patients having a median survival of $\sim 8 \pm 2$ months. ${ }^{1}$ Malignant transformation of melanocytes involves stepwise tumor progression. The early radial growth phase (RGP) advances via the vertical growth phase (VGP), into highly aggressive $\mathrm{MM}^{2,3}$ The metastatic stage still represents the major therapeutic challenge, as responders to conventional chemotherapy are still below $20 \%$ for mono- and below $30 \%$ for poly-chemotherapy. ${ }^{4-6}$ Therefore, alternative therapeutic strategies are required, and the targeting of specific activating mutations in melanoma has been of particular interest, ${ }^{7-10}$ yet almost invariably, responders develop therapy resistance. ${ }^{11}$

The majority of currently available data on human melanoma have been obtained from two-dimensional (2D) cultures of melanoma cell lines. Tumor cells, however, grow in a three-dimensional (3D) environment in which intercellular cross-talk exists between differentiated cancer cell subpopulations as well as between cancer and non-transformed neighboring host cells. Consequently, 3D skin reconstruction models have recently become attractive preclinical testing tools for novel therapeutic approaches, especially 3D models that contain human MM cells. ${ }^{12}$ However, most current models are limited to the dermal compartment of the skin that contains MM nests consisting of only few cells and therefore do not adequately recapitulate melanoma metastases. Here, we develop a novel organotypic 3D human skin and melanoma reconstruction model that closely resembles the in vivo situation of cutaneous melanoma metastasis and validate it by comparing the therapeutic effects of two TRAIL (tumor necrosis factor-related apoptosis-inducing ligand)based combination therapies.

\footnotetext{
${ }^{1}$ Institute of Cell Biology and Immunology, University of Stuttgart, 70569 Stuttgart, Germany; ${ }^{2}$ Institute for Interfacial Engineering, IGVT, University of Stuttgart, 70569 Stuttgart, Germany; ${ }^{3}$ Department of Cell and Tissue Engineering, Fraunhofer Institute of Interfacial Engineering and Biotechnology (IGB), 70569 Stuttgart, Germany; ${ }^{4}$ Viscofan BioEngineering, R\&D Biomedicals and Cell Biology, 69469 Weinheim, Germany; ${ }^{5}$ Experimental Dermatology, Department of Dermatology, TU Dresden, 01307 Dresden, Germany and ${ }^{6}$ Centre for Cell Death, Cancer and Inflammation (CCCI), UCL Cancer Institute, University College London, London, UK ${ }^{*}$ Corresponding author: D Kulms, Experimental Dermatology, Department of Dermatology, TU-Dresden, Fetscherstrasse 74, 01307 Dresden, Germany. Tel: + 49351458 18973; Fax: + 49351458 4338; E-mail: dagmar.kulms@ @uniklinikum-dresden.de

Keywords: melanoma therapy; organotypic tumor model; apoptotic cell death

Abbreviations: MM, metastatic melanoma; RGP, radial growth phase; VGP, vertical growth phase; TRAIL, tumor necrosis factor-related apoptosis-inducing ligand; UVB, ultraviolet-B radiation; XIAP, X-linked inhibitor of apoptosis protein

Received 23.4.13; revised 06.6.13; accepted 07.6.13; Edited by G Melino
} 
In a previous 2D study employing 18 cell lines derived from different tumor progression stages, we found most of the cell lines to be TRAIL resistant. ${ }^{13}$ Irradiation with sublethal ultraviolet-B radiation (UVB) synergistically rendered them TRAIL sensitive by a mechanism involving caspase-3dependent cleavage of the X-linked inhibitor of apoptosis protein $(\mathrm{XIAP}){ }^{13,14}$ Here, we find a similar, albeit less pronounced cisplatin-mediated TRAIL sensitization of melanoma cells in 2D culture. Strikingly, in the new 3D skin-melanoma model developed herein, cisplatin became substantially more active as a TRAIL sensitizer than UVB. This diametric difference in responsiveness of melanoma cells in $2 \mathrm{D}$ versus $3 \mathrm{D}$ culture to two related combinatorial treatments exemplifies the need to develop more complex preclinical model systems for human malignant melanoma. Since 2D cultures so far have failed to provide successful treatment strategies for most metastatic cancers, the 3D model may more reliably predict clinical effectiveness of novel therapeutic regimes to be taken to clinical trials. Additionally, the model provides an excellent tool to gain closer insights into intra-tumoral differentiation and tumor-host interaction.

\section{Results}

3D full-thickness skin equivalents resemble normal human skin. Successful treatment of metastasis can be influenced by both, cellular cross-talk between tumor cells and between tumor and host cells. To establish an in vivo-like environment representing human skin, we seeded primary keratinocytes on top of primary fibroblasts embedded into a collagen I scaffold. Differentiation of keratinocytes was achieved by culturing the skin equivalents at an air-liquid interface for up to 17 days. Immunohistochemical examination of the 3D full-thickness skin equivalents revealed proper epidermal stratification forming distinct layers of the epidermis as seen in normal human skin (Figures $1 \mathrm{a}$ and b). While cells from the basal epidermal layers stained positive for keratin 14, the more differentiated cells from the supra-basal layer stained positive for keratin 10 and involucrin. Highly differentiated cells close to the stratum corneum were the only ones that stained positive for filaggrin. Most importantly, laminin 5 staining revealed that, just as in normal human skin, a basal lamina was generated to physiologically connect the epidermal to the dermal portion of the artificial skin (lowest panel, Figures 1a and b). Consequently, we generated a $3 \mathrm{D}$ in vivo human skin-like environment in vitro which should prove useful to study primary dermal melanoma metastasis.

Development of a 3D full-thickness skin-melanoma metastasis model. To test whether these skin equivalents are useful to study malignant melanoma in a 3D environment, we inserted cell lines representing different progression stages. Whereas SBCL2 (RGP) and WM-115 (VGP) cells formed nest-like structures in the epidermis (black arrows) only metastatic 451-LU (MM) cells invaded deeply into the dermis to form melanoma nests (Figure 1c), indicating that the $3 D$ skin equivalent provides an organotypic environment in which melanoma cells can grow according to their progression stage.

Although skin equivalents incorporating melanoma nests could already be used to study therapeutic effectiveness, we were bothered by three shortcomings of these types of models: first, number and size of melanoma nests formed are unpredictable; second, metastases in vivo are usually larger than melanoma nests and exhibit a more complex intratumoral diversity; third, due to the limited life span of tumor-nest models, treatment is initiated early, and therefore, rather than inducing regression of existing tumor nests, it interferes with tumor outgrowth.

To overcome these limitations, we generated melanoma spheroids of defined size and cell number to be inserted at defined numbers into the organotypic skin equivalents. By culturing 250 metastatic 451-LU cells in a hanging drop for 15 days, ${ }^{15}$ we reproducibly generated spheroids consisting of viable melanoma cells presenting a compact structure with a final diameter of $\sim 500 \mu \mathrm{m}$ mimicking non-vascularized tumor nodes, micrometastasis or inter-capillary micro regions of solid tumors (Figure 2a). ${ }^{16,17}$

We next inserted a defined number of these spheroids (10) into the dermal fibroblast/collagen I scaffold at day 1 of model construction (Figure 2b), allowing them to grow and co-develop during epidermal differentiation for 17 more days. Thereby, we derived a novel 3D skin-melanoma spheroid model that contained spheroids which had been cultured in vitro for a total of 31 days in the context of artificial organotypic skin.

Melanoma spheroids integrated into skin equivalents
recapitulate key features of human cutaneous melanoma metastasis. Immunohistochemical analysis of the skin-melanoma spheroid model revealed spheroids to share many key features regarding histological appearance and cellular distribution with non-vascularized human cutaneous melanoma metastases in vivo (Figure 3). Two sub-populations of cells are clearly discernible in skinembedded melanoma spheroids (Figure 3a). The peripheral living sub-population harbors about $70 \%$ proliferating (KI-67 positive) compared with resting cells (Figure $3 b$ ), whereas the central sub-population mainly consists of shrunken, apoptotic or necrotic cells (TUNEL staining; Figure 3a), forming the 'necrotic' center. This distribution of tumor cell sub-populations is guaranteed by the spheroid sizes, being due to a lack of nutrients and oxygen in the central part where catabolic waste accumulates (Figure 3c). ${ }^{18}$ Successful treatment of melanoma is particularly impaired by the fact that metastatic disease quickly becomes resistant to chemotherapy and combination therapies, respectively. ${ }^{19,20}$ Two proteins have recently been shown to confer chemoresistance specifically to melanoma cells: the ATP-dependent drug efflux transporter $\mathrm{ABCB} 5^{21,22}$ and JARID1B, a histone $\mathrm{H} 3$ demethylase that slows down the cell cycle. ${ }^{23,24}$ Comparing the expression levels of ABCB5 and JARID1B in melanoma spheroids with primary cutaneous metastasis revealed a similar expression pattern of both proteins. In both tissues, only cells of the peripheral living populations stained positive for ABCB5 (about 20\%) and JARID1B (about $30 \%$ ), further supporting the homology of the organotypic 
a

full skin equivalent

Ш

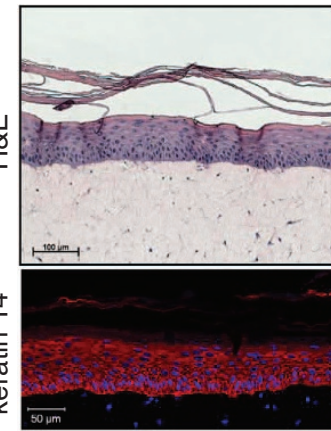

을
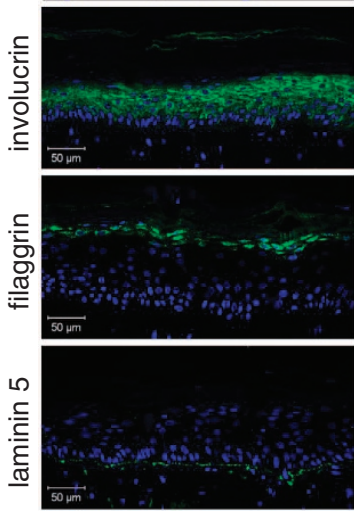

c

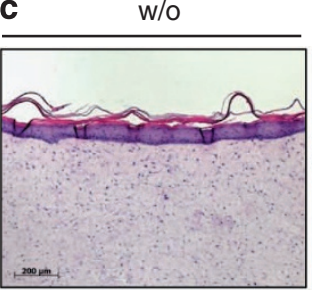

b normal human skin
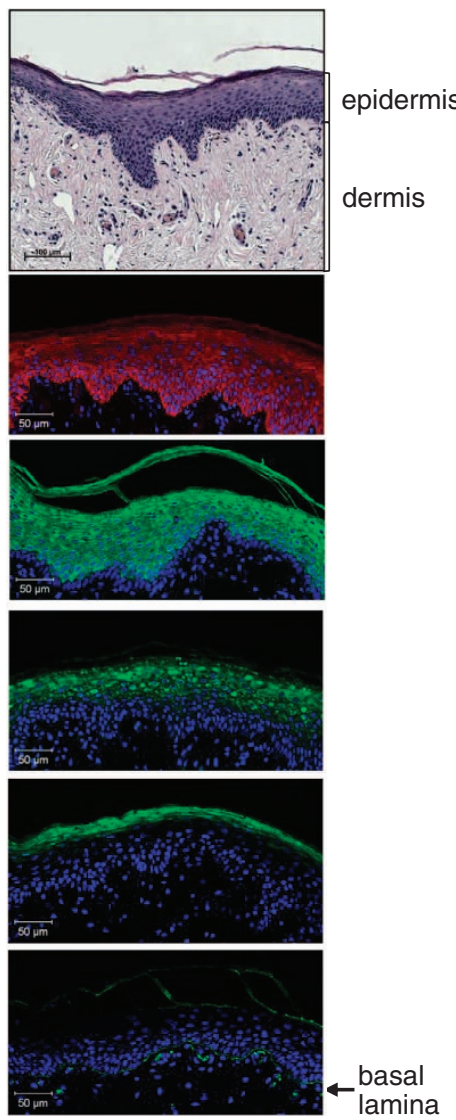
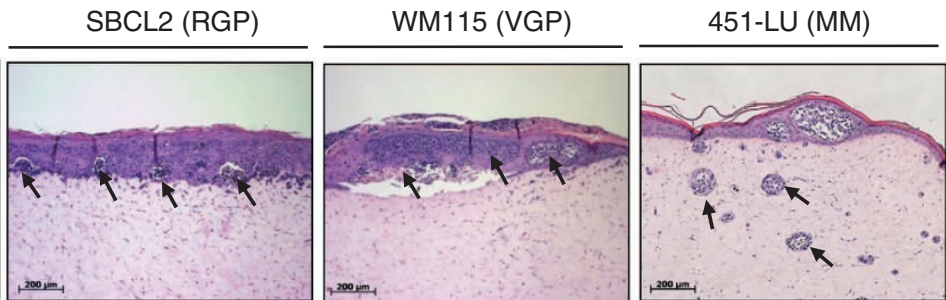

Figure 1 Generation of 3D organotypic skin equivalents. Paraffin sections of skin equivalents (a) compared with normal human skin (b) were H\&E stained and immunohistochemically analyzed for expression of keratins 14 and 10, involucrin, filaggrin and laminin 5. (c) Melanoma cells of different tumor progression stages, SBCL2 (RGP), WM115 (VGP) and 451-LU (MM), were seeded on top of the dermal layer before addition of keratinocytes. At day 17 of epidermal differentiation, the localization of melanoma cells within the reconstituted skin equivalents was visualized by H\&E staining of paraffin sections. Images show one representative out of three independently performed experiments

skin-melanoma spheroid model to in vivo metastasis (Figures 3d and e).

Major impact of melanoma environment on therapeutic response. The purpose of developing organotypic models of cancer in vitro is to provide closer insight into tumor physiology. It also allows to study both, therapeutic impact on the tumor and side effects on the surrounding host tissue, thereby serving the needs of reliable preclinical test systems. In a previous monolayer-based study, we showed that 16 out of 18 melanoma cell lines were synergistically sensitized to TRAIL-induced apoptosis by co-application of sublethal UVB radiation. ${ }^{13,14}$ Primary metastases, however, penetrate into deeper, dermal parts of the skin and secondary metastases invade even further into distant parts of the body which cannot be reached by UVB. ${ }^{25}$ We therefore aimed to identify an alternative stimulus to UVB as a TRAIL sensitizer that would engage a similar biochemical mechanism to be used systemically. Like UVB, cisplatin introduces base modifications on DNA and consequently similar physiological responses. ${ }^{26}$ Furthermore, cisplatin is known to sensitize cancer cells from tissues of various origins to TRAIL-induced apoptosis. $^{27,28}$

When used at clinically achievable concentrations, cisplatin sensitized resistant melanoma cells representing the three major progression stages SBCL2 (RGP), WM-115 (VGP) and 451-LU (MM) to TRAIL-induced apoptosis, but to a considerably lesser extent than UVB (Figure 4a). The molecular mechanism 
underlying TRAIL sensitization by cisplatin appeared similar to the one responsible for UVB-induced TRAIL sensitization. ${ }^{13,14}$ While combination with TRAIL + UVB resulted in pronounced XIAP depletion and caspase-3 processing, combination of TRAIL with cisplatin only slightly affected this process (Figure 4b).

A prerequisite for successful therapeutic strategies includes that the primary host tissue remains less affected than the tumor, or preferably unaffected. We could prove tumor selectivity for TRAIL + UVB and TRAIL + cisplatin combinations, as they did not influence viability of primary fibroblasts and melanocytes, and only slightly affected primary keratinocytes in vitro, while selectively killing 451-LU melanoma cells (Figure 4c).

Our results show that, despite sharing certain aspects with UVB-mediated TRAIL sensitization, the synergism of cisplatin-induced TRAIL sensitization of melanoma cells is less pronounced, at least in 2D cell culture. Given our intention to compare the effectiveness of different therapeutic regimes in the newly devised 3D skin-melanoma spheroid model, we reckoned that the use of two related but different therapeutic combinations could be highly informative. We therefore next compared the therapeutic effects of the TRAIL + cisplatin and TRAIL + UVB combinations in both the skin-melanoma nest and the skin-melanoma spheroid model.

Intriguingly, in the skin-melanoma nest model (Figure 4d; Supplementary Figure S1) the outcome of TRAIL + UVB versus TRAIL + cisplatin treatment was significantly different from that observed in 2D culture. Melanoma nests hosted within organotypic skin remained largely unaffected by individual treatment with TRAIL, UVB or cisplatin, but were significantly reduced in number and size when treated with TRAIL either in combination with UVB or cisplatin, both in short-term and in long-term treatment schedules (Figure 4d). Notably, whereas the tumoricidal effects of TRAIL + UVB and TRAIL + cisplatin were almost identical in long-term treatment, in short-term treatment TRAIL + cisplatin was even more potent than TRAIL + UVB (Figure 4e). In both cases, reduction in melanoma nests was mediated by selective induction of apoptosis in tumor cells because only melanoma nests that underwent combination treatment stained positive for cleaved caspase-3, while normal keratinocytes and fibroblasts were spared (Supplementary Figure S2). These results show that, while the principle of sensitization of melanoma cells to TRAIL-induced apoptosis by DNAdamaging agents translates from $2 \mathrm{D}$ to $3 \mathrm{D}$, the relative potency of both combinations significantly varied in an organotypic environment.

We next addressed how the texture of melanoma spheroids affected tumor cell sensitivity to TRAIL-UVB versus TRAILcisplatin co-treatment in vitro (Figure 5a). This analysis showed that melanoma spheroids responded to treatments combining TRAIL with UVB or cisplatin with apoptosis induction and remained unaffected by individual treatments as evidenced by AnnexinV/PI staining (Figure 5a; Supplementary Figures $\mathrm{S} 3 \mathrm{a}$ and $\mathrm{b}$ ). Intriguingly, increased

a

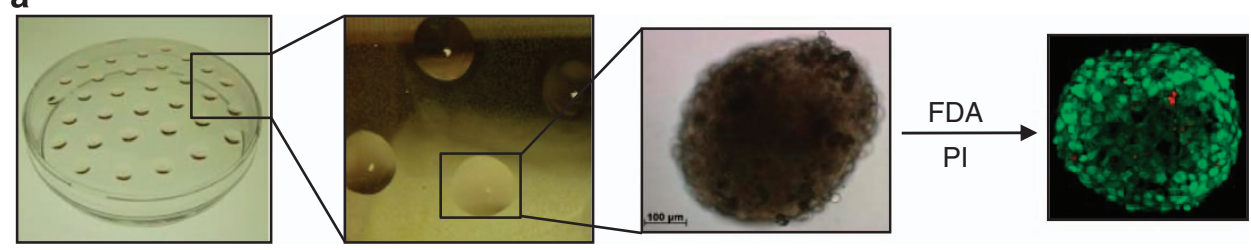

b

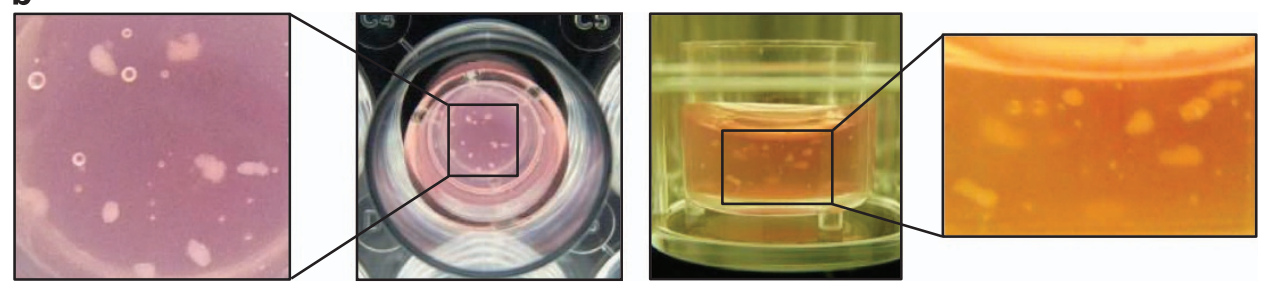

Figure 2 Generation of the organotypic melanoma-spheroid skin model. (a) 250451 -LU melanoma cells per $25 \mu$ l drop RPMI medium were placed on the lid of a non-adhesive PBS containing petri dish and incubated for 15 days deriving melanoma spheroids with a size of about $500 \mu \mathrm{m}$ in diameter. Live/death staining was performed by incubation with $5 \mu \mathrm{g}$ fluorescein diacetate (FDA) and $45 \mathrm{~g}$ propidium iodide (PI)/ml PBS for 20 min at RT in the dark. Living cells stain FDA positive (green) and dead cells stain PI positive (red). (b) Ten 451-LU spheroids were collected, carefully mixed with primary fibroblasts and added to collagen I to from the dermal compartment of the full thickness skin equivalent

Figure 3 Melanoma spheroids resemble human melanoma metastasis. (a) Paraffin sections of tumor spheroids embedded into skin equivalents were stained for proliferating (Ki67) and apoptotic (TUNEL) cells. (b) Statistical analysis of Ki67-positive cells of peripheral versus central sub-population within the spheroid. Mean \pm S.D. of three different sections of three different spheroids are shown. (c) Scheme displaying cell sub-populations within a non-vascularized spheroid/tumor. (d) Sections of untreated spheroid-containing skin equivalents and primary human cutaneous metastasis were stained for ABCB5 and JARID1B, respectively. Examples for ABCB5- or JARID1B-positive cells are indicated by black arrows. (e) Statistical analysis of ABCB5- and JARID1B-positive cells of the peripheral sub-population within the spheroid compared with human cutaneous metastasis. Mean \pm S.D. of three different sections of three different spheroids/metastasis are shown 
a

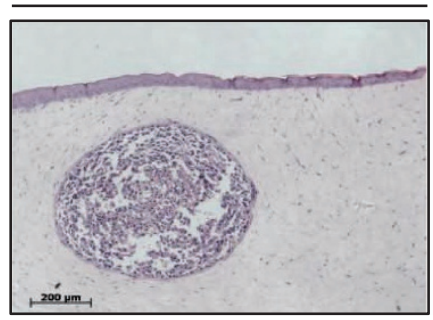

b

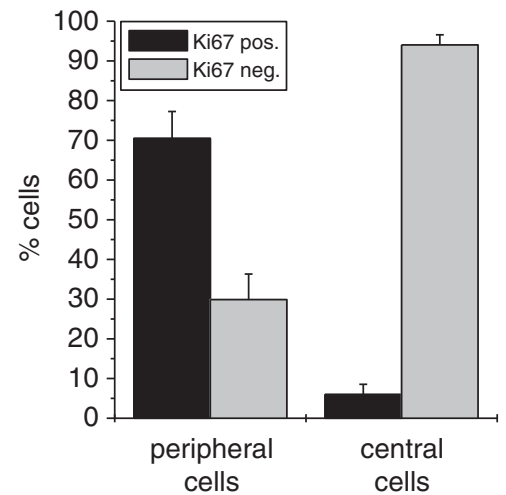

Ki67

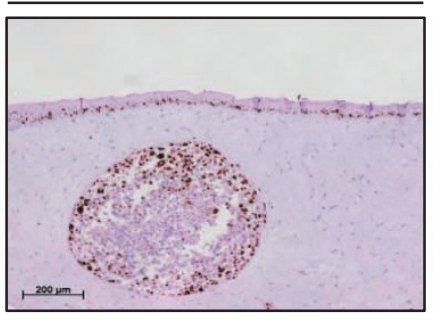

C
TUNEL
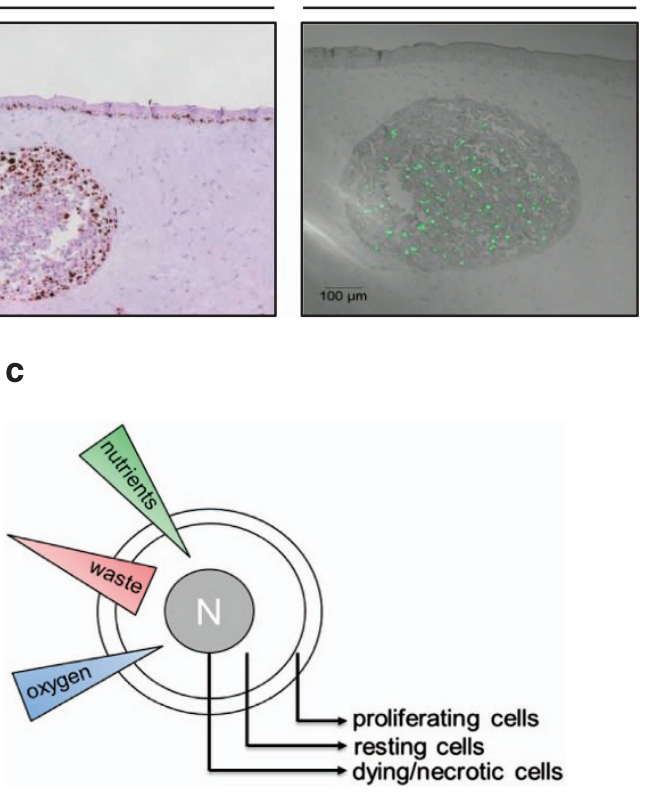

d
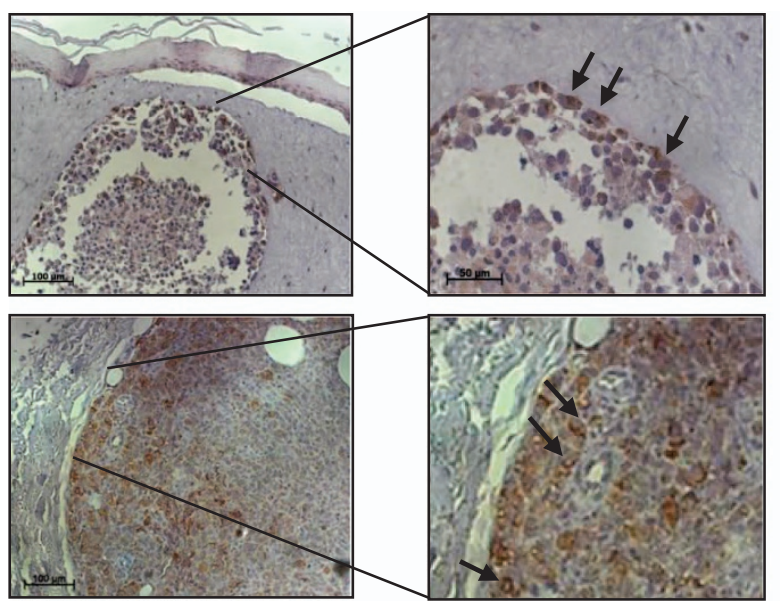
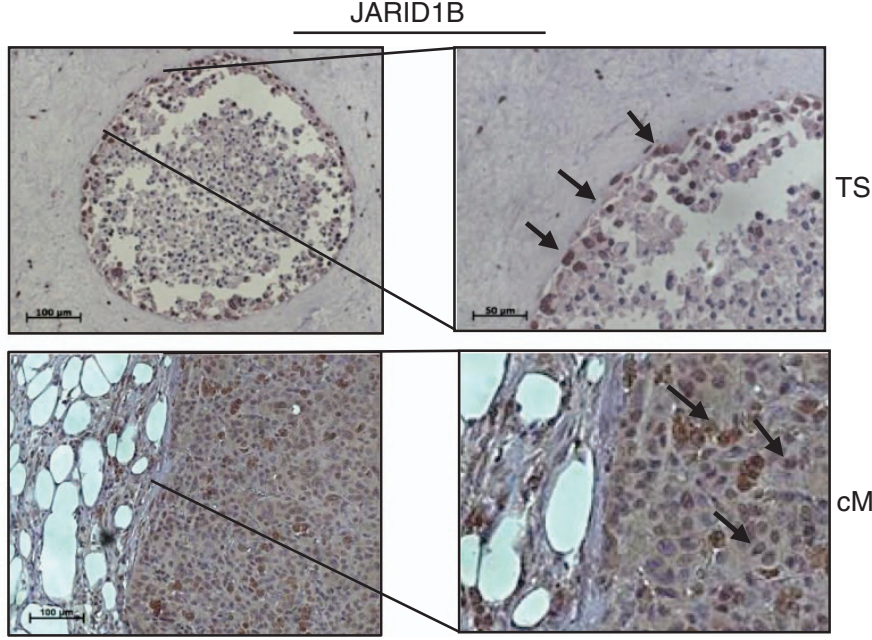

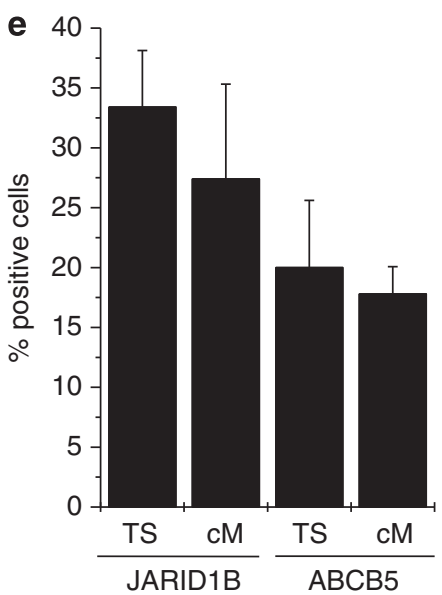


a

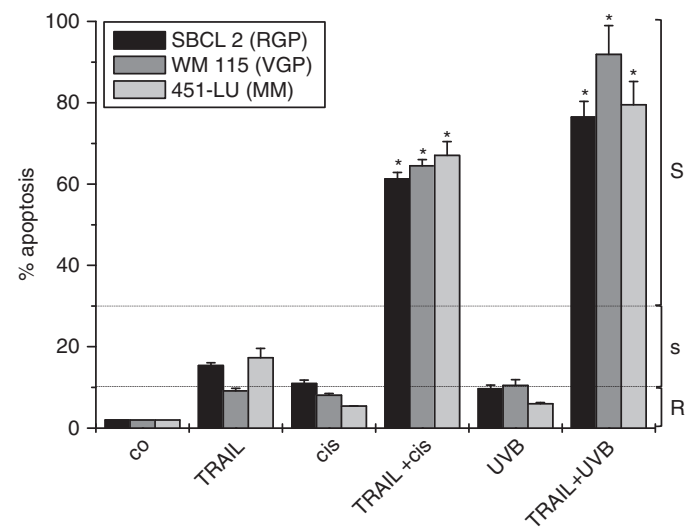

b $\frac{4}{451-\mathrm{LU}(\mathrm{s} / \mathrm{S})}$ $\begin{array}{cccccc}\text { cisplatin: }- & - & + & + & - & - \\ \text { UVB: - } & - & - & - & + & +\end{array}$
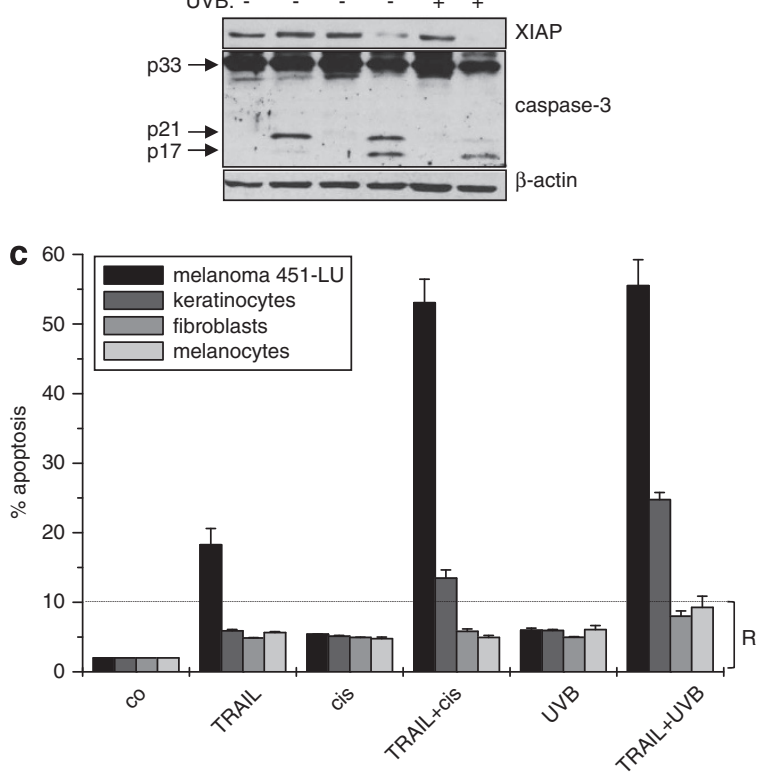

d

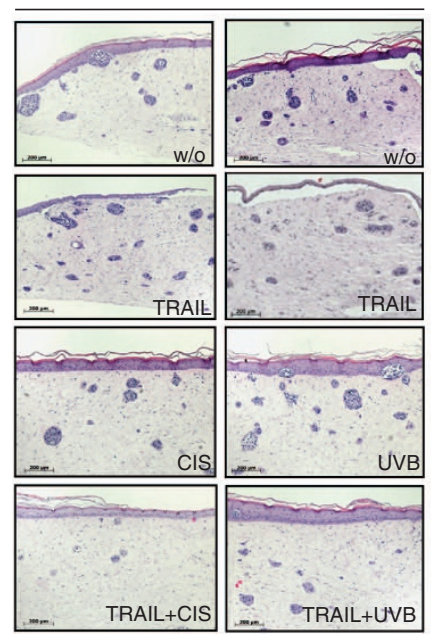

e
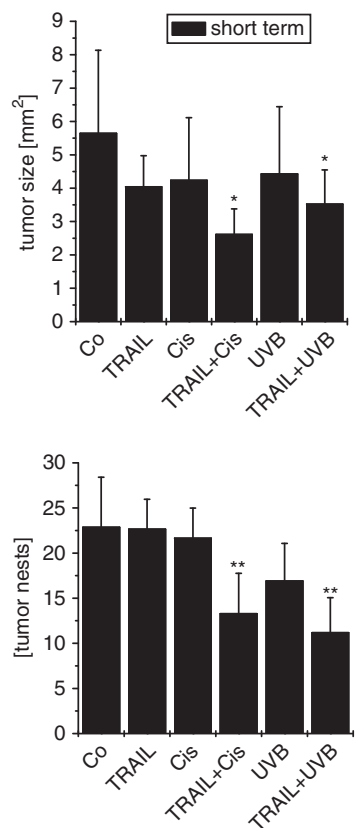
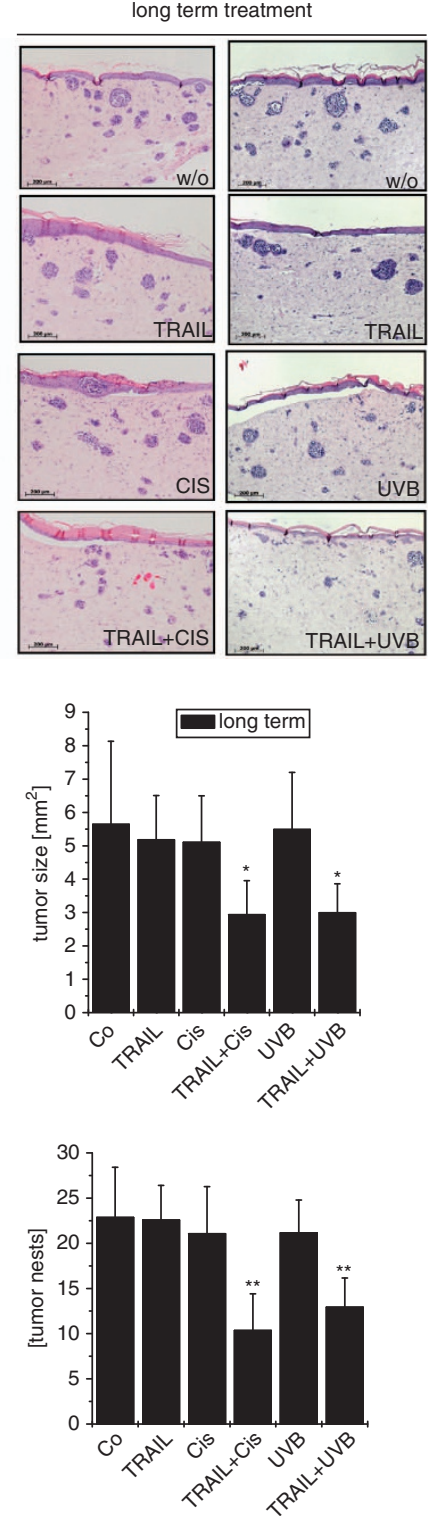

Figure 4 Combination treatment sensitizes melanoma cells but not primary cells to TRAIL. (a) SBCL2 (RGP), WM-115 (VGP) and 451-LU (MM) were stimulated with TRAIL $(100 \mathrm{ng} / \mathrm{ml})$, cisplatin $(20 \mu \mathrm{M})$ or UVB $\left(200 \mathrm{~J} / \mathrm{m}^{2}\right)$ alone, or the combination treatment. After $16 \mathrm{~h}$, apoptosis was determined using a Cell Death Detection ELISA. $[R]=$ resistant; $[\mathrm{s}]=$ slightly sensitive; $[\mathrm{S}]=$ sensitive. ${ }^{*} P<0.01$ comparing co-treated with TRAlL-only-treated cells of the same line. (b) $451-\mathrm{LU}$ cells (MM) were stimulated as in (a). After $16 \mathrm{~h}$, protein status of XIAP and cleavage pattern of caspase-3 were determined by western blot analysis. $\beta$-Actin served as loading control. One out of three experiments is shown. (c) Primary fibroblasts, keratinocytes and melanocytes as well as 451-LU melanoma cells were treated and analyzed as in (a). Results in (a) and (c) show the mean \pm S.D. of three independent experiments. (d) Skin equivalents containing 451-LU nests were subjected to either short-term treatment: TRAIL (250 $\mathrm{ng} / \mathrm{ml}$ ), cisplatin $(20 \mu \mathrm{M})$, UVB $\left(400 \mathrm{~J} / \mathrm{m}^{2}\right)$ or in combination applied twice at days 13 and 15 ; or long-term treatment: TRAIL $(250 \mathrm{ng} / \mathrm{ml})$, cisplatin $(2.5 \mu \mathrm{M})$, UVB $\left(200 \mathrm{~J} / \mathrm{m}^{2}\right)$ or in combination applied five times over 10 days. At day 17, tumor volumes determined using ImageJ software. (e) Statistical analysis of tumor nest sizes counting the 10 largest melanoma nests, and overall tumor nest numbers (mean \pm S.D.) of three sections of skin equivalents derived from five different donors. ${ }^{*} P<0.05,{ }^{* *} P<0.01$ comparing co-treated with TRAIL-only-treated tumor nests

effectiveness of TRAIL + cisplatin as compared with TRAIL + UVB in killing tumor cells was even further pronounced in melanoma spheroids, reflected by advanced $\mathrm{PI}$-positive staining of spheroids as a marker for late apoptosis/necrosis. These observations were corroborated by biochemical analysis of melanoma spheroids, which showed that TRAIL + cisplatin was substantially more effective in depleting XIAP and, consequently, enabling full processing of caspase-3 than
TRAIL + UVB (Figure 5b). This result confirmed the trend that in more complex culture systems of melanoma, the synergism of TRAIL + cisplatin becomes more pronounced whereas the one of TRAIL + UVB loses potency.

When finally analyzing the therapeutic effect of both combinations in the skin-melanoma spheroid model, we strikingly found that only TRAIL + cisplatin but not TRAIL + UVB-induced apoptosis in melanoma spheroids at all (Figure 6). 

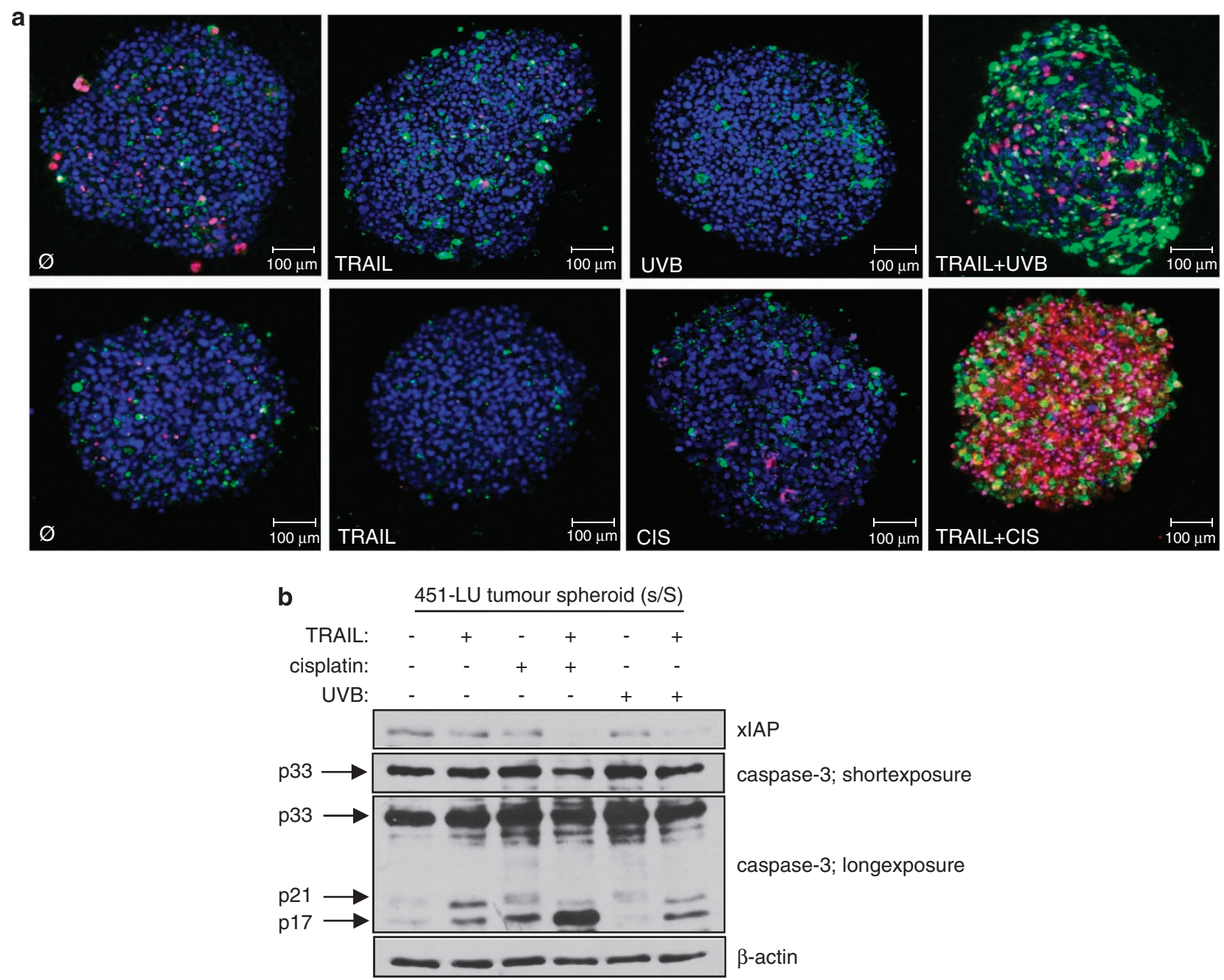

Figure 5 Melanoma spheroids respond to combination treatment in vitro. (a) Melanoma spheroids were left untreated or stimulated with TRAIL (100 $\mathrm{ng} / \mathrm{ml}$ ), cisplatin $(20 \mu \mathrm{M})$, UVB $\left(200 \mathrm{~J} / \mathrm{m}^{2}\right)$ or a combination thereof. Twenty hours later, spheroids were stained for apoptosis using FITC-labeled AnnexinV (green) and propidium iodide (Pl; red) and analyzed by immunofluorescence microscopy. Nuclei were visualized with Hoechst 33342 (blue). (b) Melanoma spheroids were treated as in (a). Sixteen hours later, the protein status of XIAP and cleavage pattern of caspase-3 were determined by western blot analysis. $\beta$-Actin served as loading control. One out of three independently performed experiments is shown

Apoptosis induction by TRAIL + cisplatin was selective for proliferating cells within the melanoma spheroids (Figures $6 \mathrm{~d}$ and f). Treatments with TRAIL, UVB and cisplatin alone, or with TRAIL + UVB, did not significantly affect cell viability (Figures $6 b, c$ and e). Importantly, the disability of UVB to sensitize melanoma cells in skin-embedded spheroids to TRAIL-induced apoptosis was not due to its insufficient tissue penetration, as cyclobutane pyrimidine dimers (CDP), generated as a result of UVB irradiation, were readily detectable even in deeper parts of the dermis (Supplementary Figure S4). TRAIL + cisplatin co-treatment resulted in almost complete disappearance of Ki67-positive melanoma cells from the peripheral sub-population of skin reconstruct-embedded melanoma spheroids, while, concomitantly, most cells stained positive for cleaved caspase-3 and TUNEL (Figure 6f). Yet, hardly any changes could be detected in spheroids following TRAIL + UVB co-treatment (Figure 6d). Like in the skinmelanoma nest model, the pro-apoptotic effect of TRAIL + cisplatin was tumor selective in the skin-melanoma spheroid model, as most primary human fibroblasts and keratinocytes survived (zoomed Figure 6f). Hence, whereas TRAIL + cisplatin exert a substantial apoptotic effect in the newly devised skin-melanoma spheroid model, TRAIL + UVB does not. Accordingly, the organotypic environment appears to influence the therapeutic outcome. These results show that diametric differences in therapeutic efficiency of two related therapeutic concepts are likely to exist between conventional 2D cell culture and more sophisticated organotypic 3D models. It furthermore offers the combination therapy of TRAIL + cisplatin to become a promising strategy to fight melanoma.

\section{Discussion}

A multitude of clinical trials conducted so far has not achieved significant benefit for patients with MM. Despite remarkable 
Ki67

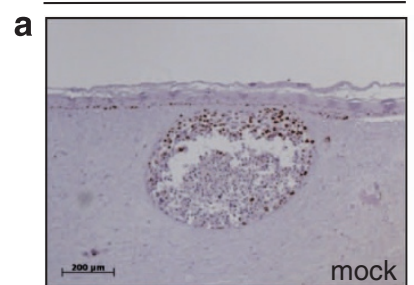

b

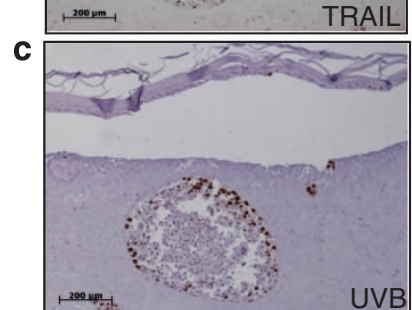

d

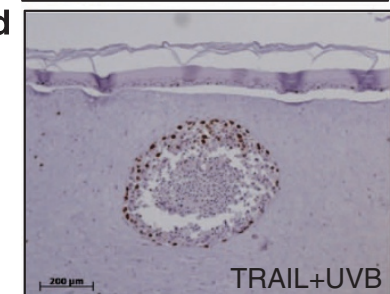

e
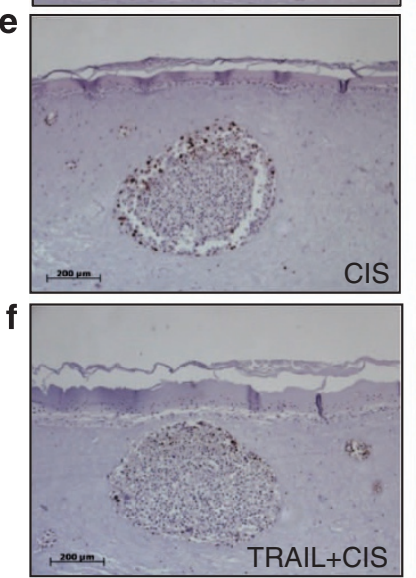

active caspase-3
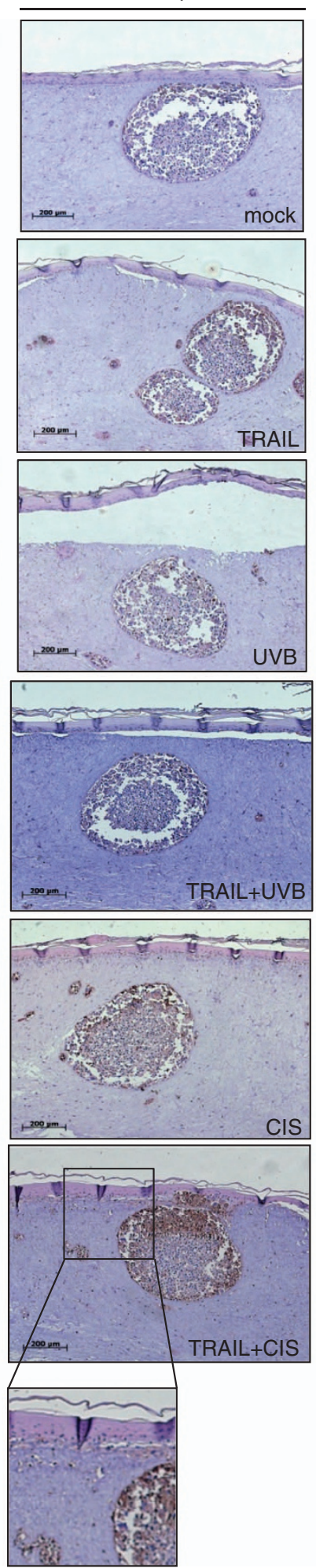

TUNEL
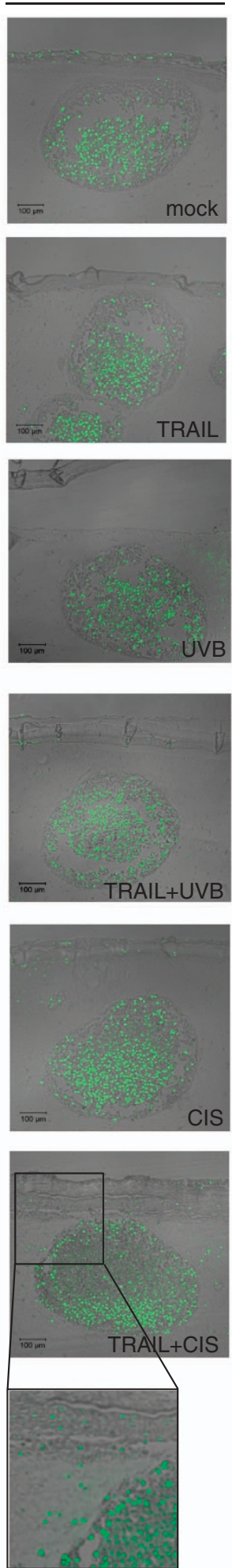

Figure 6 Melanoma spheroids integrated into skin equivalents respond to TRAIL + cisplatin but not to TRAIL + UVB. Organotypic skin equivalents containing 451-LU spheroids were left (a) untreated or subjected to short-term treatment with (b) TRAlL (500 ng/ml), (c) UVB ( $\left.400 \mathrm{~J} / \mathrm{m}^{2}\right)$ or (e) cisplatin (40 $\mu$ M) alone or in (d) TRAIL + UVB and (f) TRAIL + cispatin combination, respectively, applied twice on days 13 and 15. On day 17, paraffin sections were stained for Ki67, cleaved caspase-3 and TUNEL positivity, respectively. One representative out of three independently performed experiments is shown 
effort and promising in vitro activities, this disease remains almost invariably lethal. It therefore appears that a more thorough and reliable preclinical evaluation of novel drugs and therapeutic combination will be essential to reduce attrition rates in clinical trials and improve the benefit from therapeutic interventions. Mouse models so far have not gained substantial clinical benefit, ${ }^{29}$ because the architecture of mouse skin significantly differs from human skin. Given the need for better suitable in vitro test systems, we have generated a full thickness organotypic human skin model that displays a differentiation status homologous to normal human skin. ${ }^{30}$

Aiming to decode the molecular mechanisms decisive for melanoma resistance, we took advantage of large and differentiated tumor spheroids which, when integrated into organotypic skin reconstructs, histologically show high similarity to primary cutaneous human melanoma metastases. This includes a necrotic center and the existence of similar numbers of ABCB5- and JARID1B-expressing cells-features thought to majorly contribute to the relapse of initially responding tumors. The percentages of JARID1B- and ABCB5-positive cells counted here were higher, both in vitro and in vivo, compared with previous reports. ${ }^{24}$ However, we refer to the percentage of the vital cell population, not of the whole tumor population. The degree of cellular differentiation we observe is neither reflected in 2D cell culture nor sufficiently addressed in 3D models incorporating tumor nests. Up to a size of $150-200 \mu \mathrm{m}$ all cells contained with a spheroid can still be sufficiently supplied with nutrients via simple diffusion. Only spheroids with sizes of/above $500 \mu \mathrm{m}$ represent typical features of nonvascularized tumor tissue. ${ }^{16,31}$

To date, conventional chemotherapy-based cancer treatment is increasingly seconded by the application of targeted therapeutics, for example, inhibitors of kinases, ${ }^{32}$ the proteasome ${ }^{33}$ or NF- $\kappa \mathrm{B}$ activation, ${ }^{34}$ as well as drugs targeting apoptotic pathways, including Smac and $\mathrm{BH} 3$ mimetics $^{35}$ and agonists of apoptosis-inducing TRAIL receptors. ${ }^{36}$ Still, many melanoma cell lines were shown to be resistant against TRAILinduced apoptosis. ${ }^{13,37}$ While TRAIL + UVB was more potent at inducing apoptosis in 2D culture than TRAIL + cisplatin, increasing complexity of the screening model completely shifted the balance in favor of TRAIL + cisplatin. Importantly, in the most advanced 3D organotypic skin-melanoma spheroid model the therapeutic effect of TRAIL + UVB was virtually lost whereas the one of TRAIL + cisplatin increased substantially. Accordingly, application of this model shows that insights gained from 2D melanoma culture cannot readily be translated to complex 3D skin-melanoma models, and it implies that the tumor environment decisively impacts the response to therapy. It appears that the organotypic 3D human skin-melanoma spheroid model developed here provides an environment that more closely resembles the physiology of human malignant melanoma than previously available systems and may therefore set milestones for the study and understanding of this malignancy.

The environment of melanoma in patients is, however, even more complex; besides tumor-associated fibroblast, the microenvironment of human melanoma comprises various other non-transformed cell types, including immune cells and endothelial cells. The skin-melanoma spheroid model will serve as a basis for constructing models that integrate additional cell types present in the melanoma microenvironment, and may also be suitable to host other types of cancer. Therapeutically, the 3D skin-melanoma spheroid model will be useful for short-term screening of primary melanoma cells freshly isolated from patients. One can envisage the inclusion of spheroids derived from primary human melanoma (Supplementary Figure S5) or even of pieces of melanoma metastases. Thereby, preclinical testing of therapeutic combinations in the context of a known genetic make-up of a given melanoma (e.g., BRAF mutation) would be enabled, paving the path to more personalized approaches in melanoma therapy. In this context, an increasing complexity of the model will offer a useful platform to study the impact of soluble factors, like cytokines or RTK ligands released from tumor cells or surrounding host cells, that have been shown to influence the therapeutic outcome of melanoma. ${ }^{38}$

Including the organotypic 3D skin-melanoma model into the preclinical testing repertoire is likely to provide that only the most promising novel therapeutic concepts are taken forward into clinical testing so that, in the future, the attrition rate of potential new treatments for this disease may be reduced and their rate of success in clinical trials increased.

\section{Materials and Methods}

Cells and Reagents. Human melanoma cell lines SBCL2 (RGP), WM-115 (VGP) and 451-LU (MM) were kindly provided by Dr. Friedegund Meier, Department of Dermatology, University of Tübingen. Cells were maintained in RPMI-1640 (Life Technologies, Karlsruhe, Germany), 10\% FCS (Thermo Scientific, Langenselbold, Germany) and $1 \%$ penicillin-streptomycin (Life Technologies) at $37^{\circ} \mathrm{C}, 5 \% \mathrm{CO}_{2}$. Keratinocytes were prepared from the epidermis and fibroblasts form the dermis of juvenile human foreskin, ${ }^{39}$ Melanocytes were prepared from adult skin. ${ }^{40}$ Keratinocytes were maintained in KGM 2 (PromoCell, Heidelberg, Germany), passaged at $50-70 \%$ confluency and used at passage four. Fibroblasts were cultivated in high-glucose DMEM (Life Technologies), Melanocytes in M2 (PromoCell) and assayed in passage four. UVB irradiation was performed in PBS using a bank of six TL12 bulbs (Phillips, Eindhoven, Netherlands), emitting most of the energy within the UVB range (emission peak at $313 \mathrm{~nm}$ ). Recombinant human iz-TRAIL protein was prepared as previously described. ${ }^{41}$ Cisplatin was obtained from Platinex (Bristol, UK).

Generation and treatment of melanoma spheroids. Melanoma spheroids were generated via the 'hanging drop' method incorporating 250 melanoma cells per $25 \mu \mathrm{l}$ RPMI placed on the lid of a non-adhesive PBS containing petri dish. ${ }^{15}$ Spheroids were incubated for 15 days at $37^{\circ} \mathrm{C}, 5 \% \mathrm{CO}_{2}$. Every third day, $8 \mu \mathrm{l}$ of the medium per drop was exchanged. For in vitro stimulation, spheroids were harvested and maintained in a non-adhesive petri dish. Nuclei were stained with $1 \mathrm{mg} / \mathrm{ml}$ Hoechst 33342 (Sigma, Munich, Germany) for $30 \mathrm{~min}$ at RT. Apoptosis was determined by staining the spheroids with $0.375 \mu \mathrm{g} / \mathrm{ml}$ FITC-labeled Annexin V (MBL, Woburn, MA, USA) and $0.6 \mu \mathrm{g} / \mathrm{ml} \mathrm{PI}$ (Sigma) in binding buffer (10 mM HEPES, pH 7.4; $140 \mathrm{mM} \mathrm{NaCl} ; 2.5 \mathrm{mM} \mathrm{CaCl}$ ) for $20 \mathrm{~min}$ at RT in the dark. Stained spheroids were analyzed in CELLview dishes (Greiner-bio-one, Stuttgart, Germany) via confocal microscopy (LSM 710; Zeiss, Munich, Germany) with 1 airy aperture and a resolution of $1024 \times 1024$ pixel. Z-stacks were taken and further analyzed using ZEN 2009 smart setup software (Zeiss).

Generation of skin models. Skin models were generated using 24-well inserts (Nunclon TM $\Delta$; Nunc, Rochester, NY, USA) in 24-well plates (Greiner-bio-one). Per insert $1 \times 10^{5}$ fibroblasts in GNL (322.5 $\mathrm{ml} 2 \times$ DMEM; $7.5 \mathrm{ml} 3 \mathrm{M}$ HEPES; $1.25 \mathrm{ml}$ chondroitin-4-sulfate; $1.25 \mathrm{ml}$ chondroitin-6-sulfate; $7.5 \mathrm{ml} \mathrm{FCS}$ ) were mixed $1: 3$ with collagen I isolated from rat tails to a final volume of $500 \mu \mathrm{l}$ and cultivated in DMEM/4.5 g/l glucose $/ 1 \%$ L-glutamine $/ 10 \% \mathrm{FCS} / \mathrm{L}$ pyruvate overnight at $37^{\circ} \mathrm{C}$. Next day, dermal gels were equilibrated with $\mathrm{EGM} / 10 \%$ FCS $/ 1 \%$ PenStrep $/ 10 \mathrm{mg} / \mathrm{ml}$ gentamycine ${ }^{42}$ for $2 \mathrm{~h}$ at $37^{\circ} \mathrm{C}$. The medium was 
withdrawn and $1 \times 10^{5}$ keratinocytes in $\mathrm{EGM}^{43,44}$ carefully seeded on top and incubated for $1.5 \mathrm{~h}$ at $37^{\circ} \mathrm{C}$ to allow adhesion. Subsequently, skin equivalent was covered with EGM and cultivated for 7 days-changing the medium every other day. At day 7 , skin equivalents were transferred to 6-well plates and cultivated/ treated at the air-liquid interface in $\mathrm{MM}^{43,44}$ for 17 more days at $37^{\circ} \mathrm{C}$, changing the medium every other day. For generation of melanoma nests $10^{4} 451-\mathrm{LU}$ cells were seeded onto the dermal layer $1 \mathrm{~h}$ before keratinocytes were added. Alternatively, 10 tumor spheroids were added to fibroblasts containing GNL and mixed with collagen I as above.

Immunohistochemical analyses of organotypic skin equivalents. Skin models were fixed in Roti-Histofix (Roth, Karlsruhe, Germany) for $3 \mathrm{~h}$ at RT released from the insert and embedded into paraffin. Sections of $3 \mu \mathrm{m}$ were cut using an RM 2145-microtome (Leica, Biberach, Germany), transferred onto slides (LABOnord; Greiner-bio-one) for hematoxylin-eosin (HE) staining or onto sialynized slides (Menzel GmbH, Braunschweig, Germany) for immunohistochemical analysis and dried at $37^{\circ} \mathrm{C}$ overnight. Sections were released from paraffin using Roticlear (Roth) and subjected to HE staining at RT or were incubated with primary antibodies against caspase-3 (\#9446; Cell Signaling, Beverly, MA, USA), S100B (330M-15; Cell Marque, Rocklin, CA, USA), ABCB5 (Sigma), Ki67, keratin 10 (Dako, Hamburg, Germany), keratin 14, laminin 5 (Santa Cruz, Heidelberg, Germany), filaggrin (Biomedia, Singapore) and involucrin (Acris, Herford, Germany), respectively, as recommended by the manufacturer at $4^{\circ} \mathrm{C}$ overnight. For peroxidase detection, anti-mouse or anti-rabbit antibodies (EnVision; Dako) were incubated for $30 \mathrm{~min}$ at RT and liquid $D A B+$ substrate chromogen substrate (Dako) added for up to $10 \mathrm{~min}$ at RT. Slides were fixed with Isomount 2000 (LABOnord) and analyzed microscopically (Axiovert 200M, AxioCam HRc; Zeiss). For immunofluorescence analysis, secondary polyclonal goat-anti-mouse-FITC (Dako) or goat-anti-mouse-Cy3 IgG, Newmarket, UK (Jackson ImmunoResearch, Newmarket, UK) antibodies were used, slides mounted in ProLong Gold with or without DAPI (Molecular Probes Life Technologies, Darmstadt, Germany) and analyzed using a confocal microscope (LSM 710, ZEN 2009 software; Zeiss) or fluorescence microscope for spheroids (Zeiss Axiovert 200M, Axio vision rel. 4.8 software; Zeiss). TUNEL staining was performed using an In Situ Cell Death Detection Kit (Roche; Mannheim, Germany). Sections from human cutaneous metastasis were kindly provided by the Clinic of Dermatology, University of Dresden and treated as above. Volumes of melanoma nests were determined using ImageJ (NIH) software, calculating the 10 largest nests of individual sections.

Determination of cell death. Apoptosis was measured using a Cell Death Detection ELISA kit (Roche). The enrichment of mono- and oligo-nucleosomes released into the cytoplasm is calculated as: absorbance of sample cells/ absorbance of control cells. The enrichment factor of 2 corresponds to $10 \%$ apoptotic cells as determined by AnnexinV-FITC staining and FACS analysis.

Western blot analysis. Cells were lysed in lysis buffer $(50 \mathrm{mM}$ Hepes, pH 7.5; $150 \mathrm{mM} \mathrm{NaCl} ; 10 \%$ glycerol; $1 \%$ Triton X-100; $1.5 \mathrm{mM} \mathrm{MgCl} ; 1 \mathrm{mM}$ EGTA; $100 \mathrm{mM} \mathrm{NaF} ; 10 \mathrm{mM}$ pyrophosphate, $0.01 \% \mathrm{NaN}_{3}$ and Complete, Roche), tumor spheroids were disrupted by sonication in lysis buffer and the protein content was determined by DC Bio-Rad Protein assay kit (Bio-Rad, Hercules, CA, USA). In all, $60-80 \mu \mathrm{g}$ of protein samples was subjected to $13-15 \%$ SDS-PAGE, and western blot analysis using antibodies directed against caspase-3, (\#9662; Cell Signaling), XIAP (\#610716; BD Biosciences, Heidelberg, Germany) or $\beta$-actin (Sigma). Protein bands were visualized with HRP-conjugated secondary antibodies (Amersham, Buckinghamshire, UK) in a chemiluminescent reaction (SuperSignal; PIERCE, Rockford, IL, USA).

\section{Conflict of Interest}

HW declares the following conflict of interest: Inventor of TRAIL-R2specific antibodies; founder of Apogenix, a biotech company that develops apoptosis-related therapeutics.

Acknowledgements. We thank Dr. Stein, Department of Dermatology, University of Dresden for kindly providing sections of human cutaneous metastasis. We also thank S Thude for technical support at the Department of Cell and Tissue Engineering (IGB), Stuttgart. The present work was funded by the German Cancer Foundation (Deutsche Krebshilfe: 108826).
1. Balch CM, Gershenwald JE, Soong SJ, Thompson JF, Atkins MB, Byrd DR et al. Final version of 2009 AJCC melanoma staging and classification. J Clin Oncol 2009 27: 6199-6206.

2. Clark WH Jr. Human cutaneous malignant melanoma as a model for cancer. Cancer. Metastasis Rev 1991; 10: 83-88.

3. Elder DE, Clark WH Jr., Elenitsas R, Guerry D, Halpern AC. The early and intermediate precursor lesions of tumor progression in the melanocytic system: common acquired nevi and atypical (dysplastic) nevi. Semin Diagn Pathol 1993; 10: 18-35.

4. Chapman PB, Einhorn LH, Meyers ML, Saxman S, Destro AN, Panageas KS et al. Phase III multicenter randomized trial of the Dartmouth regimen versus dacarbazine in patients with metastatic melanoma. J Clin Oncol 1999; 17: 2745-2751.

5. Bleehen NM, Newlands ES, Lee SM, Thatcher N, Selby P, Calvert AH et al. Cancer Research Campaign phase II trial of temozolomide in metastatic melanoma. J Clin Oncol 1995; 13: 910-913.

6. Keilholz U, Goey SH, Punt CJ, Proebstle TM, Salzmann R, Scheibenbogen C et al. Interferon alfa-2a and interleukin-2 with or without cisplatin in metastatic melanoma: a randomized trial of the European Organization for Research and Treatment of Cancer Melanoma Cooperative Group. J Clin Oncol 1997; 15: 2579-2588.

7. Dai DL, Martinka M, Li G. Prognostic significance of activated Akt expression in melanoma: a clinicopathologic study of 292 cases. J Clin Oncol 2005; 23: 1473-1482.

8. Meier F, Schittek B, Busch S, Garbe C, Smalley K, Satyamoorthy K et al. The RAS/RAF/ MEK/ERK and PI3K/AKT signaling pathways present molecular targets for the effective treatment of advanced melanoma. Front Biosci 2005; 10: 2986-3001.

9. Satyamoorthy K, Li G, Gerrero MR, Brose MS, Volpe P, Weber BL et al. Constitutive mitogen-activated protein kinase activation in melanoma is mediated by both BRAF mutations and autocrine growth factor stimulation. Cancer Res 2003; 63: 756-759.

10. Chapman PB, Hauschild A, Robert C, Haanen JB, Ascierto P, Larkin J et al. Improved survival with vemurafenib in melanoma with BRAF V600E mutation. N Engl J Med 2011; 364: 2507-2516.

11. Flaherty KT, Puzanov I, Kim KB, Ribas A, McArthur GA, Sosman JA et al. Inhibition of mutated, activated BRAF in metastatic melanoma. N Engl J Med 2010; 363: 809-819.

12. Meier F, Busch S, Lasithiotakis K, Kulms D, Garbe C, Maczey E et al. Combined targeting of MAPK and AKT signalling pathways is a promising strategy for melanoma treatment. Br J Dermatol 2007; 156: 1204-1213.

13. Thayaparasingham B, Kunz A, Peters N, Kulms D. Sensitization of melanoma cells to TRAIL by UVB-induced and NF-kappaB-mediated downregulation of xIAP. Oncogene 2009; 28: 345-362.

14. Hornle M, Peters N, Thayaparasingham B, Vorsmann H, Kashkar H, Kulms D. Caspase-3 cleaves XIAP in a positive feedback loop to sensitize melanoma cells to TRAIL-induced apoptosis. Oncogene 2011; 30: 575-587.

15. Foty R. A simple hanging drop cell culture protocol for generation of 3D spheroids. J Vis Exp 2011; 51, pii 2720.

16. Hirschhaeuser F, Menne H, Dittfeld C, West J, Mueller-Klieser W, Kunz-Schughart LA. Multicellular tumor spheroids: an underestimated tool is catching up again. J Biotechnol 2010; 148: 3-15.

17. Sutherland RM, Durand RE. Growth and cellular characteristics of multicell spheroids. Recent Results Cancer Res 1984; 95: 24-49.

18. Zabierowski SE, Herlyn M. Melanoma stem cells: the dark seed of melanoma. J Clin Oncol 2008; 26: 2890-2894.

19. Eigentler TK, Caroli UM, Radny P, Garbe C. Palliative therapy of disseminated malignant melanoma: a systematic review of 41 randomised clinical trials. Lancet Oncol 2003; 4: 748-759.

20. Mouawad R, Sebert M, Michels J, Bloch J, Spano JP, Khayat D. Treatment for metastatic malignant melanoma: old drugs and new strategies. Crit Rev Oncol Hematol 2010; 74: 27-39.

21. Frank NY, Pendse SS, Lapchak PH, Margaryan A, Shlain D, Doeing $C$ et al. Regulation of progenitor cell fusion by ABCB5 P-glycoprotein, a novel human ATP-binding cassette transporter. J Biol Chem 2003; 278: 47156-47165.

22. Frank NY, Margaryan A, Huang Y, Schatton T, Waaga-Gasser AM, Gasser M et al. ABCB5-mediated doxorubicin transport and chemoresistance in human malignant melanoma. Cancer Res 2005; 65: 4320-4333.

23. Roesch A, Mueller AM, Stempfl T, Moehle C, Landthaler M, Vogt T. RBP2-H1/JARID1B is a transcriptional regulator with a tumor suppressive potential in melanoma cells. Int J Cancer 2008; 122: 1047-1057.

24. Roesch A, Fukunaga-Kalabis M, Schmidt EC, Zabierowski SE, Brafford PA, Vultur A et al. A temporarily distinct subpopulation of slow-cycling melanoma cells is required for continuous tumor growth. Cell 2010; 141: 583-594.

25. Kulms D, Schwarz T. 20 years after-milestones in molecular photobiology. $J$ Investig Dermatol Symp Proc 2002; 7: 46-50.

26. Strozyk E, Poppelmann B, Schwarz T, Kulms D. Differential effects of NF-kappaB on apoptosis induced by DNA-damaging agents: the type of DNA damage determines the final outcome. Oncogene 2006; 25: 6239-6251.

27. Belyanskaya LL, Marti TM, Hopkins-Donaldson S, Kurtz S, Felley-Bosco E, Stahel RA. Human agonistic TRAIL receptor antibodies Mapatumumab and Lexatumumab induce apoptosis in malignant mesothelioma and act synergistically with cisplatin. Mol Cancer 2007; 6: 66. 
28. Vondalova BO, Jelinkova I, Szoor A, Skender B, Soucek K, Horvath V et al. Cisplatin and potent platinum(IV) complex-mediated enhancement of TRAIL-induced cancer cells killing is associated with modulation of upstream events in the extrinsic apoptotic pathway. Carcinogenesis 2011; 32: 42-51.

29. Seok J, Warren HS, Cuenca AG, Mindrinos MN, Baker HV, Xu W et al. Genomic responses in mouse models poorly mimic human inflammatory diseases. Proc Natl Acad Sci USA 2013; 110: 3507-3512

30. Breitkreutz D, Mirancea N, Nischt R. Basement membranes in skin: unique matrix structures with diverse functions? Histochem Cell Biol 2009; 132: 1-10.

31. Lin RZ, Chang HY. Recent advances in three-dimensional multicellular spheroid culture for biomedical research. Biotechnol J 2008; 3: 1172-1184.

32. Woyach JA, Johnson AJ, Byrd JC. The B-cell receptor signaling pathway as a therapeutic target in CLL. Blood 2012; 120: 1175-1184.

33. Kapoor P, Ramakrishnan V, Rajkumar SV. Bortezomib combination therapy in multiple myeloma. Semin Hematol 2012; 49: 228-242.

34. Chen DJ, Huerta S. Smac mimetics as new cancer therapeutics. Anticancer Drugs 2009 20: $646-658$

35. Curiel-Lewandrowski C, Swetter SM, Einspahr JG, Hsu CH, Nagle R, Sagerman P et al. Randomized, double-blind, placebo-controlled trial of sulindac in individuals at risk for melanoma: evaluation of potential chemopreventive activity. Cancer 2012; 11: 5848-5856.

36. Falschlehner C, Ganten TM, Koschny R, Schaefer U, Walczak H. TRAIL and other TRAIL receptor agonists as novel cancer therapeutics. Adv Exp Med Biol 2009; 647: 195-206.

37. Nguyen $T$, Zhang XD, Hersey P. Relative resistance of fresh isolates of melanoma to tumor necrosis factor-related apoptosis-inducing ligand (TRAIL)-induced apoptosis. Clin Cancer Res 2001; 7: 966s-973s.

38. Wilson TR, Fridlyabd J, Yan Y, Penuel E, Burton L, Chan E et al. Widespread potential for growth-factor-driven resistance to anticancer kinase inhibitors. Nature 2012; 487: 505-509.
39. Aasen T, Izpisua Belmonte JC. Isolation and cultivation of human keratinocytes from skin or plucked hair for the generation of induced pluripotent stem cells. Nat Protoc 2010; 5: 371-382.

40. Edmondson PC, Curley RK, Marsden RA, Robinson D, Allaway SL, Willson CD. Screening for malignant melanoma using instant photography. J Med Screen 1999; 6 : 42-46.

41. Ganten TM, Koschny R, Sykora J, Schulze-Bergkamen H, Buchler P, Haas TL et al. Preclinical differentiation between apparently safe and potentially hepatotoxic applications of TRAIL either alone or in combination with chemotherapeutic drugs. Clin Cancer Res 2006; 12: 2640-2646.

42. Meier F, Nesbit M, Hsu MY, Martin B, Van Belle P, Elder DE et al. Human melanoma progression in skin reconstructs: biological significance of bFGF. Am J Pathol 2000; 156: 193-200.

43. Chen CS, Lavker RM, Rodeck U, Risse B, Jensen PJ. Use of a serum-free epidermal culture model to show deleterious effects of epidermal growth factor on morphogenesis and differentiation. J Invest Dermatol 1995; 104: 107-112.

44. Satyamoorthy K, Meier F, Hsu MY, Berking C, Herlyn M. Human xenografts, human skin and skin reconstructs for studies in melanoma development and progression. Cancer Metastasis Rev 1999; 18: 401-405.

(c) (i) () $\Theta$ Cell Death and Disease is an open-access journal published by Nature Publishing Group. This work is licensed under the Creative Commons Attribution-NonCommercial-No Derivative Works 3.0 Unported License. To view a copy of this license, visit http://creativecommons.org/licenses/by-nc-nd/3.0/

Supplementary Information accompanies this paper on Cell Death and Disease website (http://www.nature.com/cddis) 